Results A significant positive correlation between TCBF and CVO $(r=0.81$ and $\mathrm{P}<0.001)$ was seen. The TCBF $(20.21$ $\pm 4.58 \mathrm{ml} / \mathrm{s}$ versus $11.78 \pm 2.03 \mathrm{ml} / \mathrm{s} ; \quad \mathrm{P}<0.001)$ and $\mathrm{CVO}$ $(12.80 \pm 3.82 \mathrm{ml} / \mathrm{s}$ versus $9.03 \pm 2.31 \mathrm{ml} / \mathrm{s} ; \mathrm{P}=0.010)$ were significantly higher in children compared to adult volunteers. The CVO/TCBF ratio was significantly lower in children versus adult volunteers $(0.63 \pm 0.01$ versus $0.76 \pm 0.02$, $\mathrm{P}=0.025)$. In adults, the correlation of TCBF with age remains strong ( rho $=-0.69$, t-stat $=-4.5, \mathrm{P}=0.00018$ ). However, CVO (rho $=-0.29$, t-stat $=-1.42, \mathrm{P}=0.171$ ) and $\mathrm{CVO} / \mathrm{TCBF}$ ratio $(\mathrm{r}=0.16, \mathrm{P}=0.446)$ were not significantly associated with age in the adult cohort. The ratio of cerebral arterial inflow to systemic aortic outflow was significantly higher in children compared to adults $(0.45 \pm 0.08$ versus 0.15 $\pm 0.02, \mathrm{P}<0.001)$.

Conclusions Both TCBF and CVO decrease with age, however unlike TCBF, there is no correlation between the decrease in CVO through the Transverse sinuses and age, which could suggest the early development of alternative venous drainage pathways through the emissary and extracranial veins. This could also explain the differential ratio of CVO to TCBF, which suggests that more than $20 \%$ of cerebral venous outflow in adults and more than $35 \%$ of outflow in Children are not through the Transverse sinuses in the supine position. Understanding the quantitative differences between TCBF and CVO in healthy volunteers could help identify and manage changes related to venous outflow abnormalities.

Disclosures R. Abdalla: None. S. Schnell: None. M. Aristova: None. M. Hurley: None. S. Ansari: None. A. Shaibani: None.

\section{E-174 RECOGNITION OF SAH PATIENTS AT HIGH RISK FOR SYMPTOMATIC VASOSPASM: RESPONSES TO ACETAZOLAMIDE CHALLENGE}

${ }^{1} \mathrm{D}$ Dawkins*, ${ }^{2} \mathrm{E}$ Harvey, ${ }^{2} \mathrm{~K} \mathrm{Li},{ }^{3} \mathrm{~B}$ Aagaard-Kienitz, ${ }^{1} \mathrm{D}$ Niemann, ${ }^{1} \mathrm{M}$ Baskaya, ${ }^{4} \mathrm{~S}$ Schafer, ${ }^{5} \mathrm{C}$ Strother, ${ }^{1} \mathrm{~A}$ Ahmed. ${ }^{1}$ Neurosurgery, University of Wisconsin, Madison, WI; ${ }^{2}$ Medical Physics and Radiology, University of Wisconsin, Madison, WI; ${ }^{3}$ Neurosurgery and Radiology, University of Wisconsin, Madison, Wl; ${ }^{4}$ Research Collaborations Manager Siemens Medical Solutions, Siemens Healthineers, Malvern, PA; ${ }^{5}$ Radiology, University of Wisconsin, Madison, WI

\subsection{6/neurintsurg-2020-SNIS.206}

Introduction Cerebral vasospasm can be a devastating sequela of aneurysmal SAH. There is neither a satisfactory predictor nor a standardized protocol for its early detection. We evaluated cone beam CT perfusion (CBCTP) with acetazolamide challenge as a potential tool to detect deficiencies in autoregulation prior to development of symptomatic vasospasm.

Methods Ten patients presenting with aneurysmal SAH within 24 hours of symptom onset were enrolled in this pilot study. Just prior to the initial DSA study, a baseline CBCTP acquisition was performed (60 cc contrast, $60 \mathrm{cc}$ saline). Acetazolamide (1 g IV) was administered and, after a 20-minute delay, a second CBCTP acquisition was done. Data was then reconstructed into perfusion maps (CBF, CBV, and MTT). Delay insensitive deconvolution was used and AIFs were manually selected. Motion correction was applied if possible. Data was analyzed for percent change in perfusion maps in response to acetazolamide, variation between cerebral hemispheres, and study related complications. Percent change in CBF with a $p$ value $<0.05$ was defined as significant.

Results Perfusion maps were successfully acquired for all 10 patients. Our analysis was performed on 20 cerebral hemispheres (right and left). Nineteen hemispheres demonstrated a significant change in $\mathrm{CBF}$ in response to acetazolamide $(p<0.05)$. Six hemispheres demonstrated a $30 \%$ change in CBF and 12 had a 15\% change. In 9 hemispheres there was an appropriate increase in $\mathrm{CBF}$ in response to acetazolamide. In 10 hemispheres there was a decrease in CBF. There was no statistical difference in the percent change between left and right hemispheres which would correlate with the global vasodilatory effects of acetazolamide. Symptomatic vasospasm developed in 2 patients. One vasospasm patient had a pathological decrease in $\mathrm{CBF}$ in response to acetazolamide while the other had an appropriate increase in CBF. There were no study related complications.

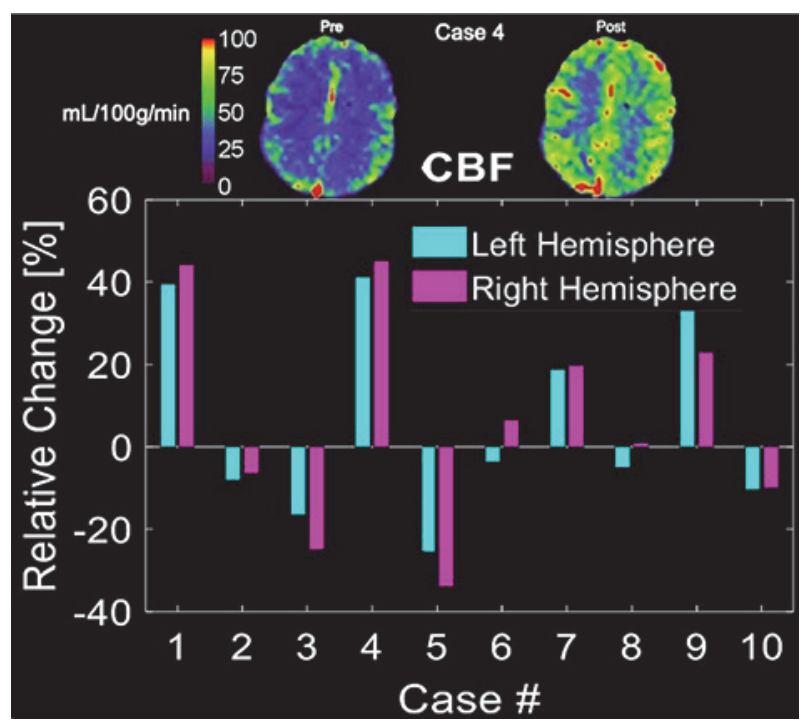

Abstract E-174 Figure 1 Relative percent change in CBF calculated for 10 patients ( 20 hemispheres). Positives percent change correlates with increase in $\mathrm{CBF}$ and negative with decrease in CBF. Perfusion maps pre and post acetazolamide demonstrate qualitative increase in CBF for case 4

Conclusion Accurate early identification of patients at high risk for symptomatic vasospasm would be a valuable clinical tool in the management of aneurysmal SAH. This small pilot study shows the feasibility of CBCTP with acetazolamide challenge to generate reproducible, diagnostic quality perfusion maps with a response to acetazolamide. These provide the opportunity to evaluate the vasodilatory capacity or state of autoregulation at a time before vasospasm is angiographically or symptomatically evident. A larger study is ongoing to provide data which will help to better understand the significance of changes observed in our study.

Disclosures D. Dawkins: None. E. Harvey: None. K. Li: None. B. Aagaard-Kienitz: None. D. Niemann: None. M. Baskaya: None. S. Schafer: 1; C; Siemens Research Support Multi-phasic acquisition and reconstruction under development. Not available for sale in the U.S.A.. C. Strother: None. A. Ahmed: 2; C; Siemens. 